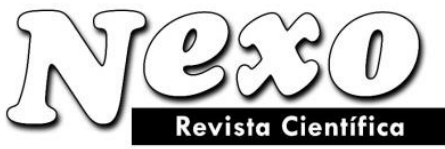

ISSN-E 1995-9516

Universidad Nacional de Ingeniería COPYRIGHT (C) (UNI). TODOS LOS DERECHOS RESERVADOS http://revistas.uni.edu.ni/index.php/Nexo https://doi.org/10.5377/nexo.v34i06.13120

\title{
Patrón de distribución temporal de la lluvia en la estación aeropuerto de Managua, período 2002-2016
}

\section{Time distribution pattern of rainfall at Managua airport station, period 2002- 2016}

\author{
Miguel E. Blanco Chávez \\ Universidad Nacional de Ingeniería. \\ Programa de Investigación, Estudios Nacionales y Servicios del Ambiente. Managua, Nicaragua. \\ miguel.blanco@piensa.uni.edu.ni
}

(recibido/received: 22-septiembre-2021; aceptado/accepted: 28-octubre-2021)

\begin{abstract}
RESUMEN
En el presente artículo se aborda el patrón de distribución de la lluvia en la estación pluviográfica Aeropuerto de Managua. El objetivo del estudio fue la determinación de los hietogramas de lluvia y los coeficientes de avance de la tormenta en cada Cuartil, en el período 2002-2016. Se estimaron las curvas masas acumuladas de precipitación de las tormentas con altura mayor que $20 \mathrm{~mm}$ y duraciones menores o iguales a dos h. Las curvas masas de precipitación fueron normalizadas con respecto a su duración y altura de precipitación. Posteriormente, se obtuvieron los patrones de distribución de la lluvia para las tormentas con altura mayor que $20 \mathrm{~mm}$ y para tormentas mayores que $30 \mathrm{~mm}$, los cuales podrían ser utilizados en las estimaciones de caudales máximos para determinar crecidas en el área de influencia de la estación Aeropuerto de Managua. El estudio sugiere que el patrón de distribución de la lluvia obtenido en ambos casos, no es simétrico y difiere del obtenido con el método de los bloques alternos.
\end{abstract}

Palabras claves: Hietograma; Cuartil; Pluviograma; Método de los Bloques Alternos; Probabilidad de la lluvia.

\section{ABSTRACT}

In the present paper is exposed the study of the rainfall patter distribution at Airport Managua pluviograph station. The study objective was determining the rainfall hyetographs and the advanced coefficients for the storm at each quartile, in the period 2002-20016. The rainfall accumulated curve mass were estimated for the storms with depth greater than $20 \mathrm{~mm}$ and duration less or equal to two $\mathrm{h}$. The rainfall accumulated curve mass were normalized with respect theirs duration and precipitation depth. Forward, the rainfall distribution pattern were obtained for the storms with depth greater than $20 \mathrm{~mm}$ and for the storms with depth greater than $30 \mathrm{~mm}$, which could be used for the estimates of maximum discharges to determines floods at Managua airport station influence area. The study suggests that the rainfall distribution patter obtained en both cases, it is not symmetric and differs from that obtained with the alternate blocks method.

Keywords: Hyetograph; Quartile; Pluviograph; Alternating Block Method; Rainfall probability 


\section{INTRODUCCIÓN}

En las estimaciones de caudales máximos de crecidas se utilizan patrones de lluvia característicos de la zona de estudio, pero desafortunadamente, en muchos casos no se dispone de estos datos y los calculistas utilizan métodos que la bibliografía especializada ofrece, los cuales fueron obtenidos para otras latitudes, dando como resultado en muchos casos, sobreestimaciones. Así, se dispone de tormentas de diseño, que "... son una herramienta muy empleada para los estudios hidrológicos, en concreto aquellos relacionadas con las crecidas, ya que permiten obtener una lluvia sintética con la cual reducir la incertidumbre." (Balbastre et al, 2018, p. 16 ).

Por otro lado, Chow et al (1988) menciona que las tormentas de diseño se utilizan como entrada de los modelos de simulación hidrológica ya que su transformación mediante procedimientos lluvia escorrentía permite conocer los caudales por lo que son empleados en el diseño de estructuras hidráulicas.

Las características de la tormenta de diseño según Watt et al (1986), se muestran en la Figura 1, las cuales son:

- Duración de la tormenta, td. En términos generales representa el tiempo transcurrido entre el inicio y fin de la lluvia.

- Intervalo de discretización de tormenta $\Delta$ t. Es el intervalo de tiempo en el que se divide la duración de la tormenta.

- Intensidad de lluvia, i. Es la cantidad de precipitación por unidad de tiempo.

- Intensidad máxima de lluvia, Imáx. Es el valor máximo de la cantidad de precipitación por unidad de tiempo.

- Volumen de lluvia acumulado por intervalo de tiempo $\Delta \mathrm{t}$, v.

- Volumen total acumulado V.

- Tiempo en el que se alcanza el pico de intensidad de la tormenta, tp.

Debemos agregar el coeficiente de avance de la tormenta $r$, que es la relación del tiempo en que se presenta la intensidad máxima al tiempo de duración de la tormenta.

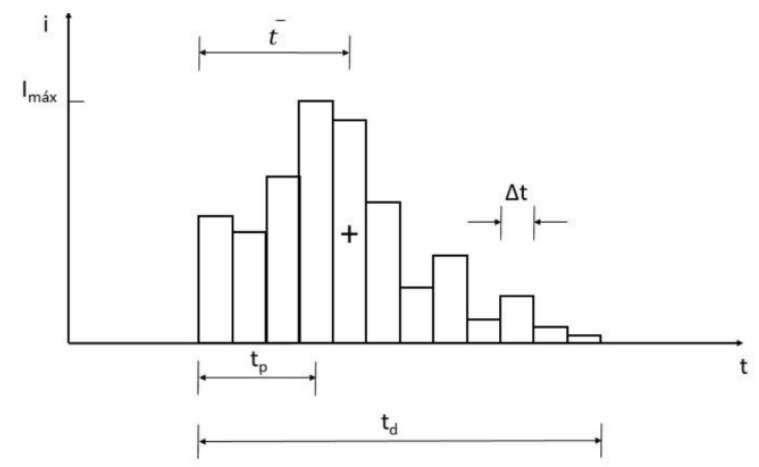

Figura 1. Representación esquemática de las características de una tormenta Fuente: Adaptado de (Watt, et al., 1986).

Para poder definir esta tormenta de diseño (hietograma), se han formulado varios métodos a partir de las curvas IDF y otros a partir de los registros de precipitación observada. 
Entre los diferentes métodos de obtención de tormentas sintéticas de diseño, uno de los más difundidos es el método de los bloques alternos (Chow et al, 1988), el cual se basa en las curvas IDF representativa de la zona de estudio. Consiste en elegir una curva IDF para un determinado período de retorno o recurrencia de la tormenta, y para intervalos consecutivos del tiempo $\Delta \mathrm{t}$, hasta un tiempo igual al tiempo de duración de la tormenta $\mathrm{t}_{\mathrm{d}}=\mathrm{n} \Delta \mathrm{t}$, se obtienen las intensidades y las alturas de precipitación como el producto de la intensidad por la duración, luego se calculan las diferencias de alturas de los intervalos consecutivos y se ordenan colocando la mayor en el centro, y las otras se alternan a la derecha y a la izquierda del bloque central, obteniendo así un hietograma sintético simétrico. El coeficiente de avance de la tormenta es $r=$ 0.5 , indicando que la máxima intensidad se presenta a la mitad de la duración de la tormenta.

El Servicio de Conservación de Suelos (SCS), hoy Servicio de Conservación de Recursos Naturales (Natural Resources Conservation Services [NRCS], 1986), también desarrolló hietogramas sintéticos a partir de tormentas observadas en Estados Unidos de América para tormentas de seis a 24 h de duración, clasificándolas según la zona del país. Para que estos hietogramas sean utilizados en otros países, debenser validados en la zona de aplicación. Respecto al coeficiente de avance de la tormenta, "... en muchos lugares se ha obtenido que las tormentas presentan un coeficiente menor que 0.5 , presentando el pico de la intensidad avanzado respecto a la duración total." (Balbastre et al, 2018, p. 28).

El hietograma de diseño de la Encuesta del agua del estado de Illinois (en inglés Illinois State Water Survey, ISWS) del Instituto de Investigación de la Universidad de Illinois. La metodología para obtener la distribución temporal se basa en un trabajo previo de F.A. Huff en el cual se estudiaron fuertes tormentas de tres horas de duración y superiores, registradas en zonas que abarcan áreas de hasta 103600 hectáreas de la parte central de Illinois (Huff, 1967). Los autores Balbastre et al, I (2018, p. 40) mencionan que "Del análisis estadístico de estos datos, el autor encontró una relación entre la duración de la tormenta y el cuartil donde ocurre la mayor parte de la precipitación...", así, para tormentas con duraciones menores de $12 \mathrm{~h}$, la mayor parte de la lluvia ocurre en los cuartiles uno y dos, para tormentas entre 12 y $24 \mathrm{~h}$, la mayor parte de la lluvia ocurre en el cuartil tres, y para tormentas mayores de $24 \mathrm{~h}$, en el cuartil cuatro (Mays, 2001).

En el estado de Santa Catarina, Brasil, Back, A.J. (2011), desarrollo un estudio de la distribución temporal de eventos de lluvias intensas, en base a tormentas observadas, obteniendo resultados similares a los de Huff (1967), lo cual confirma que las condiciones locales son importantes para decidir cual modelo de hietograma sintético utilizar.

En la subcuenca sur del lago Xolotlán, no se dispone del perfil de distribución temporal de la lluvia, con, que permita obtener hietogramas sintéticos de diseño. El objetivo de este estudio fue obtener los patrones de distribución temporal de la lluvia de corta duración, para tormentas con altura total mayor que 20 y 30 $\mathrm{mm}$ respectivamente y duración menor o igual a dos horas.

\section{METODOLOGÍA Y DESARROLLO}

Se seleccionó la estación Aeropuerto de Managua por ser la que tiene las series de precipitación más larga, está clasificada como hidrometeorológica principal (HMP) y dispone de pluviogramas. Está ubicada en las coordenadas $12^{\circ} 8^{\prime} 36^{\prime \prime}$ de latitud norte y $86^{\circ} 9^{\prime} 49^{\prime \prime}$ de longitud oeste, es parte de la subcuenca sur del Lago Xolotlán en el pacífico de Nicaragua, y según la clasificación Pfafstetter (Instituto Nicaragüense de Estudios Territoriales [INETER], 2014) pertenece a la cuenca 9525 (cuenca 69 en el sistema PHCA) o cuenca de los grandes lagos de Nicaragua que drena al mar Caribe por el río San Juan.

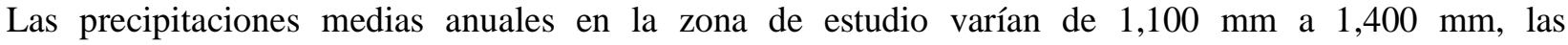
temperaturas medias anuales varían de 28 a $24{ }^{\circ} \mathrm{C}$, la evapotranspiración potencial media anual varía de 
los 1,800 a 2,000 mm, la humedad relativa media anual varía entre 70 a $75 \%$, el índice de confort climático anual se clasifica como Muy cálido opresivo (MCo) y la clasificación climática según Koppen modificado es Clima caliente y subhumedo con lluvias en verano AW (INETER, 2004).

\subsection{Procesamiento de datos}

Los gráficos de los pluviogramas diarios fueron digitalizados y almacenados a escala de cinco minutos, y se utilizó la hoja de cálculo Excel para el manejo de los archivos de datos, selección y clasificación de los eventos extremos. Los eventos de tormentas se clasificaron en cuatro tipos, determinando la cantidades precipitadas en los cuatro cuartiles de duración de la lluvia (Huff, 1967). La tormenta es categorizada por la duración que tiene la mayor precipitación: la lluvia es clasificada como tipo I si la mayoría de la lluvia ocurre en el primer $25 \%$ de la duración total; como tipo II si la mayoría de la lluvia ocurre entre el 25 y el $50 \%$ de su duración; como tipo III si la mayoría de la lluvia ocurre entre el 50 y el $75 \%$ de su duración; y como tipo IV si la mayoría de la lluvia ocurre en el último $25 \%$ de su duración total. Se analizaron de forma separada los eventos de lluvia de cada tipo, determinando los porcentajes acumulados con intervalos de 5\%, hasta $100 \%$ de su duración total. Para cada duración, se determinó el porcentaje de la serie respecto a la lluvia total.

Tormentas con duración menor o igual a 120 minutos y con altura de precipitación mayor o igual a 20 $\mathrm{mm}$ : Se procesaron 104 pluviogramas de tormentas en el período 2002-20016. En la Tabla 1 se muestra la cantidad de eventos de lluvia por cuartil, en la cual se observa que el cuartil II contiene la mayor cantidad de los eventos, seguidos de los cuartiles I, III y IV. Los cuartiles I y II contienen $82 \%$ del total de los eventos, el cuartil IV contiene la menor cantidad de eventos, apenas el 2\%. La Figura 2 muestra la distribución porcentual de las tormentas analizadas por cuartil.

Tabla 1.Localización de la intensidad máxima.

\begin{tabular}{ccc}
\hline Cuartil & Cantidad & $\%$ \\
\hline I & 34 & 33 \\
II & 51 & 49 \\
III & 17 & 16 \\
IV & 2 & 2 \\
\hline Total & 104 & 100 \\
\hline
\end{tabular}

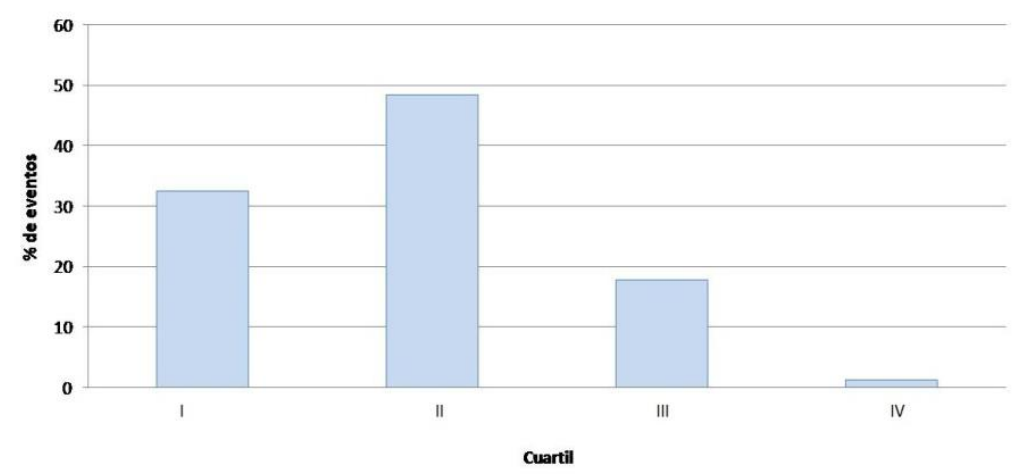

Figura 2. Distribución porcentual por cuartil de las tormentas analizadas 
Las alturas de precipitación de las tormentas analizadas variaron entre $20 \mathrm{~mm}$ y $120 \mathrm{~mm}$, su distribución porcentual se muestra en la Figura 3. Los eventos más frecuentes varían entre 20 y $30 \mathrm{~mm}$ de altura. El 74 $\%$ de los eventos tienen una $\mathrm{P}<40 \mathrm{~mm}$ y $87 \%$ de los eventos tienen una $\mathrm{P}<50 \mathrm{~mm}$.

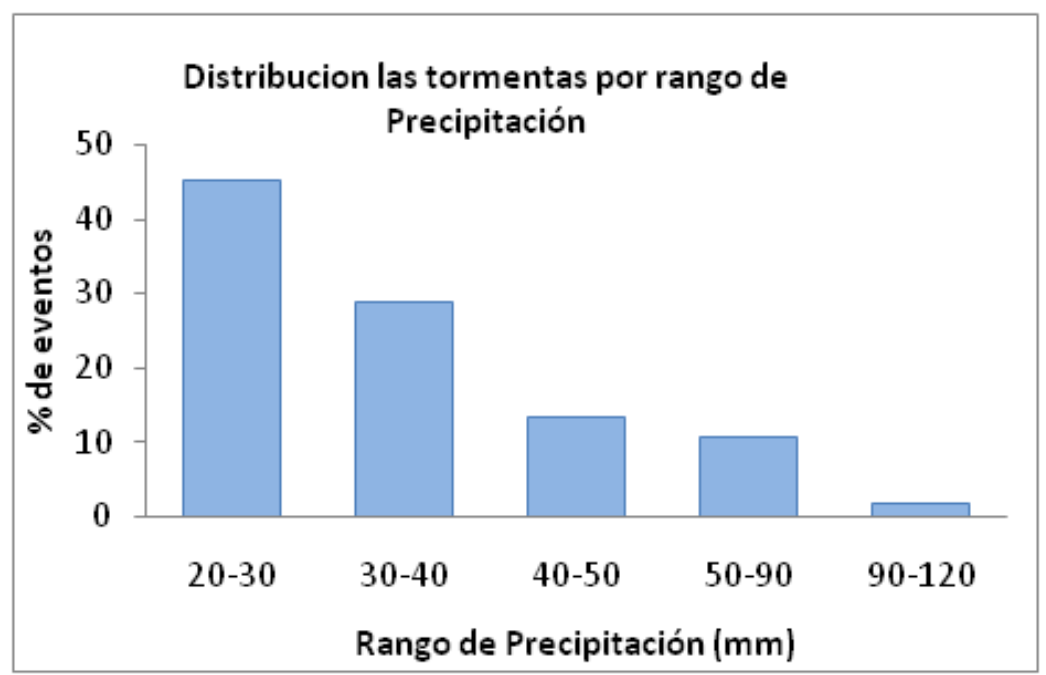

Figura 3: Distribución de las tormentas por rango de precipitación

Se analizó el patrón de distribución temporal de la lluvia para los eventos agrupados por cuartil. Las probabilidades de ocurrencia de los eventos son mayores para los cuartiles 1 y 2 ( $82 \%$ juntos), apenas $16 \%$ para el cuartil 3 y solo $2 \%$ para el cuartil 4. La Tabla 2 muestra los coeficientes de distribución temporal de la tormenta para cada cuartil.

\begin{tabular}{ccccc}
\multicolumn{5}{c}{ Tabla 2: Patrón de distribución de la lluvia para Td<=2 h y P $>=20 \mathrm{~mm}}$. \\
\hline \multirow{2}{*}{ \% T acum } & $\begin{array}{c}\text { C-I } \\
\text { \% P Acum }\end{array}$ & $\begin{array}{c}\text { C-II } \\
\text { \% P Acum }\end{array}$ & $\begin{array}{c}\text { C-III } \\
\text { \% P Acum }\end{array}$ & $\begin{array}{c}\text { C-IV } \\
\text { \% P Acum }\end{array}$ \\
\hline 0 & 0 & 0 & 0 & 0 \\
5 & 4.8 & 2.1 & 1.3 & 1.2 \\
10 & 14.3 & 6.2 & 4.0 & 3.2 \\
15 & 24.3 & 11.0 & 7.0 & 5.7 \\
20 & 34.6 & 16.4 & 10.2 & 8.3 \\
25 & 44.6 & 22.9 & 13.7 & 11.0 \\
30 & 53.0 & 30.7 & 17.5 & 14.5 \\
35 & 60.3 & 39.4 & 21.6 & 18.3 \\
40 & 66.6 & 48.5 & 26.5 & 22.3 \\
45 & 72.5 & 57.5 & 32.8 & 26.4 \\
50 & 77.8 & 66.3 & 40.4 & 30.9 \\
55 & 81.4 & 72.8 & 49.2 & 35.9 \\
60 & 84.6 & 78.8 & 57.7 & 42.4 \\
65 & 87.6 & 83.6 & 65.6 & 49.5 \\
70 & 90.3 & 87.6 & 72.8 & 57.0 \\
75 & 92.7 & 91.0 & 79.5 & 65.0 \\
80 & 94.5 & 93.7 & 85.5 & 73.5 \\
85 & 96.1 & 95.9 & 90.6 & 84.4 \\
90 & 97.5 & 97.4 & 94.6 & 91.7 \\
95 & 98.8 & 98.7 & 97.5 & 97.4 \\
100 & 100.0 & 100.0 & 100.0 & 100.0 \\
\hline
\end{tabular}


La Figura 4 muestra para cada cuartil las curvas de distribución promedio de la lluvia en el tiempo, y las figuras 5, 6,7 y 8 muestran los hietogramas desarrollados para cada cuartil, cada uno ilustra el porcentaje de la lluvia total para incrementos del $10 \%$ de la duración de la tormenta.

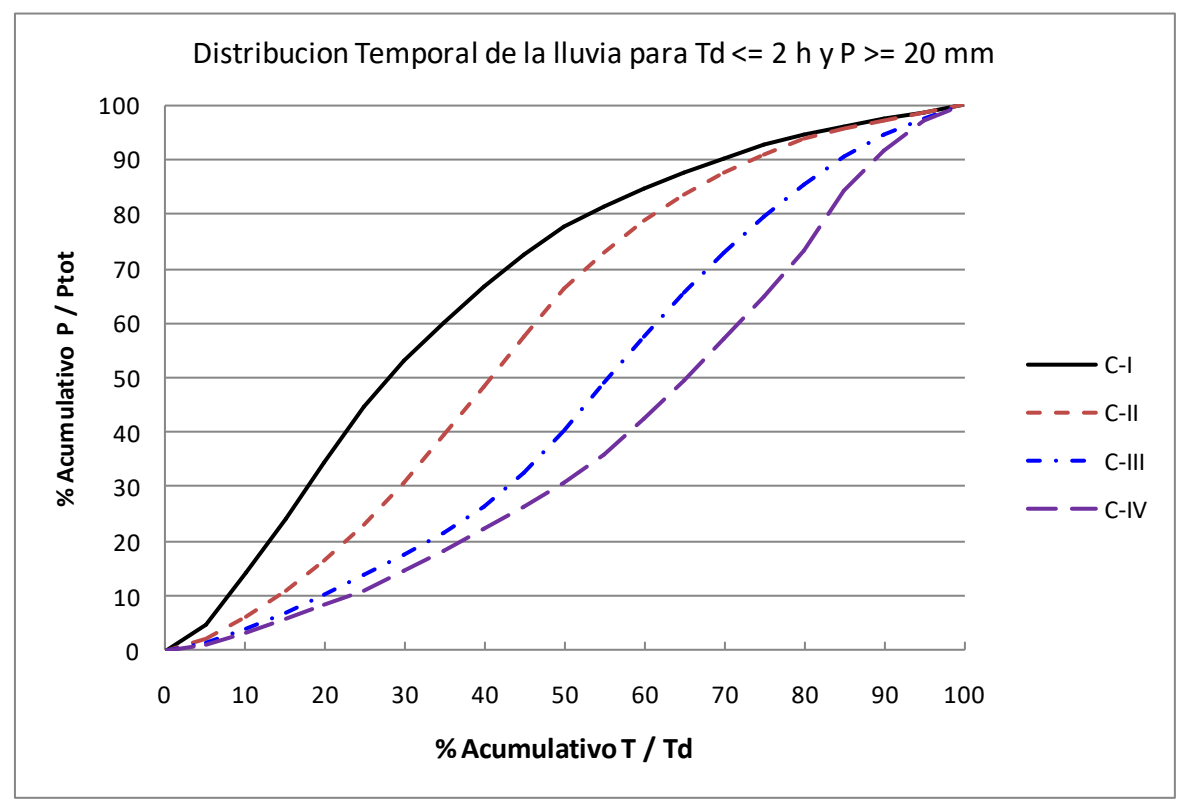

Figura 4: Curvas de distribución temporal de la lluvia para $\mathrm{Td}<=2$ h y $\mathrm{P}>=20 \mathrm{~mm}$

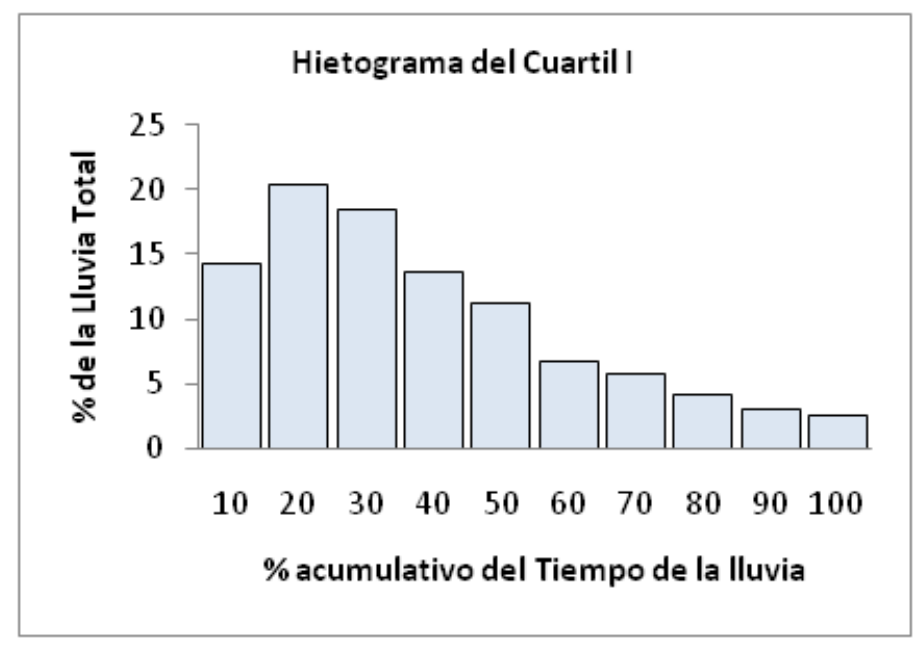

Figura 5: Hietograma del Cuartil I para $\mathrm{Td}<=2 \mathrm{~h}$ y $\mathrm{P}>=20 \mathrm{~mm}$ 


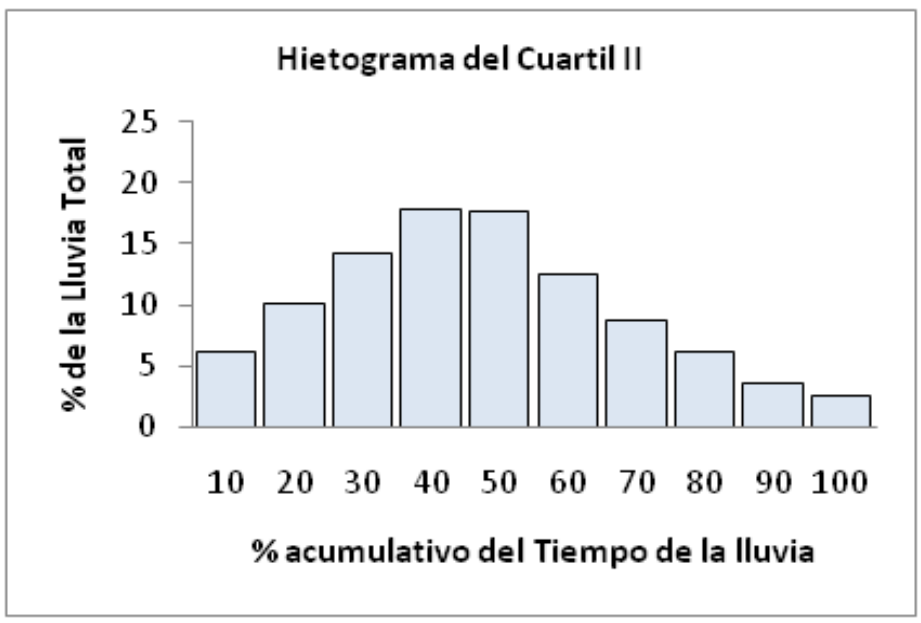

Figura 6: Hietograma del Cuartil II para $\mathrm{Td}<=2 \mathrm{~h}$ y P $>=20 \mathrm{~mm}$

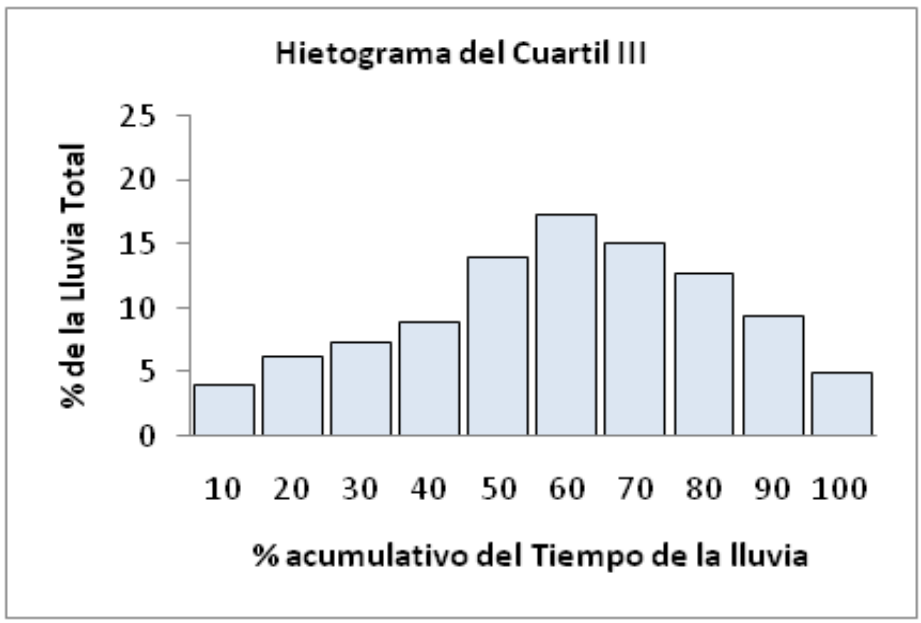

Figura 7: Hietograma del Cuartil III para $\mathrm{Td}<=2 \mathrm{~h}$ y $\mathrm{P}>=20 \mathrm{~mm}$

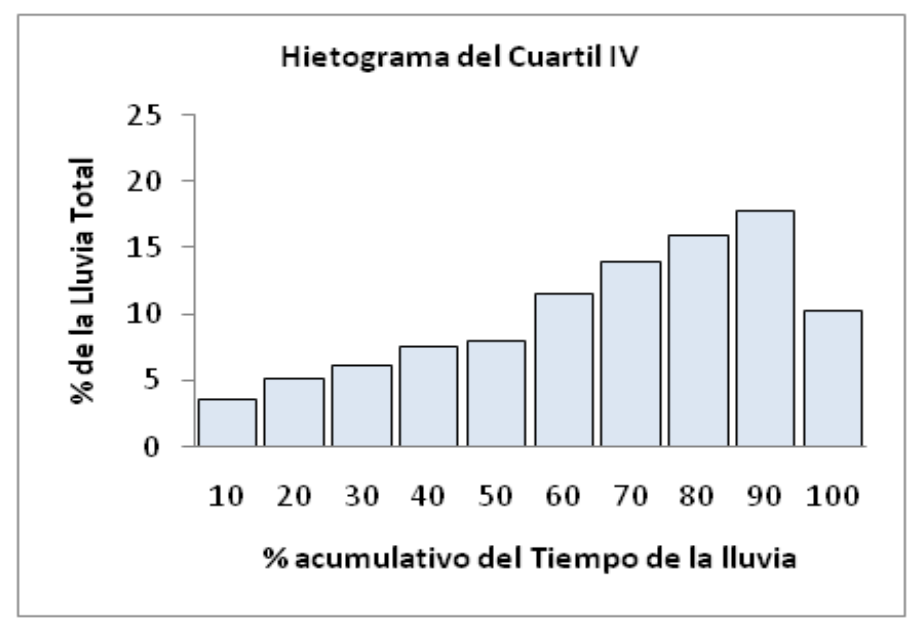

Figura 8: Hietograma del Cuartil IV para $\mathrm{Td}<=2 \mathrm{~h}$ y $\mathrm{P}>=20 \mathrm{~mm}$ 
De los hietogramas anteriores se obtiene los coeficientes de avance de las tormentas $r$, para el cuartil I es 0.15 , para el cuartil II es 0.35 , para el cuartil III es 0.55 y para el cuartil IV es 0.85 .

Tormentas con duración menor o igual a 120 minutos con altura de precipitación mayor o igual a 30 mm: Se procesaron un total de 59 tormentas en el período 2002-20016. En la Tabla 3 se muestra la cantidad de eventos de lluvia por cuartil, en la cual se observa que el cuartil II contiene la mayor cantidad de los eventos, seguidos de los cuartiles I, III y IV. Los cuartiles I y II contienen $84 \%$ del total de los eventos, el cuartil IV contiene la menor cantidad de eventos, apenas el 3\%. La Figura 9 muestra la distribución porcentual de las tormentas analizadas por cuartil.

Tabla 3.Localización de la Intensidad Máxima.

\begin{tabular}{ccc}
\hline Cuartil & Cantidad & $\%$ \\
\hline I & 18 & 30.5 \\
II & 32 & 54.2 \\
III & 7 & 11.9 \\
IV & 2 & 3.4 \\
\hline Total & 59 & 100 \\
\hline
\end{tabular}

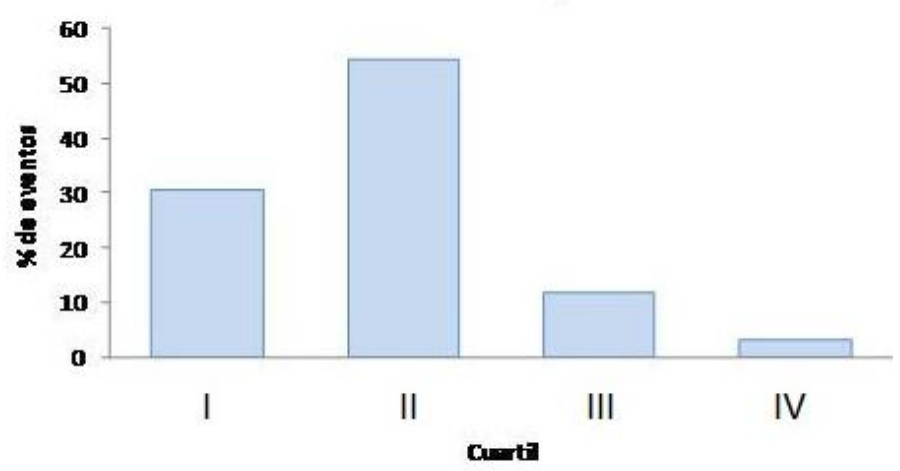

Figura 9: Distribución porcentual por cuartil de las tormentas analizadas

Las alturas de precipitación de las tormentas analizadas variaron entre $30 \mathrm{~mm}$ y $120 \mathrm{~mm}$, su distribución porcentual se muestra en la Figura 10. Los eventos más frecuentes varían entre 20 y $30 \mathrm{~mm}$ de altura. El $54 \%$ de los eventos tienen una $\mathrm{P}<40 \mathrm{~mm}$ y $78 \%$ de los eventos tienen una $\mathrm{P}<50 \mathrm{~mm}$.

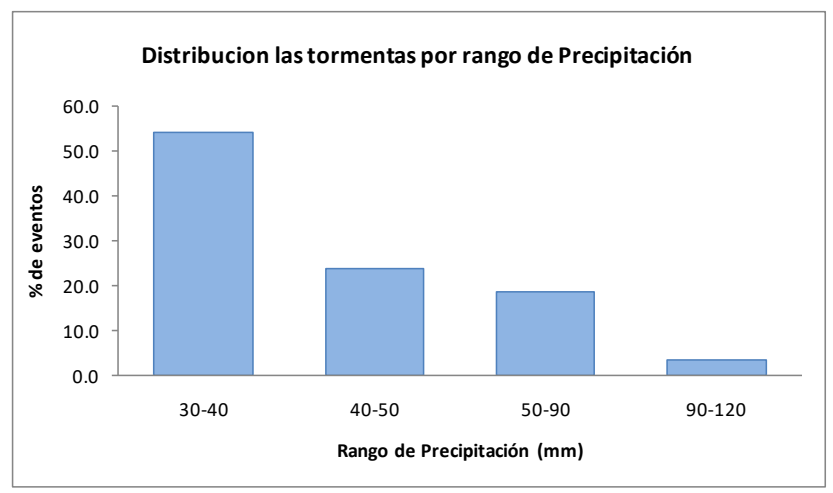

Figura 10:Distribución de las tormentas por rango de precipitación 
Se analizó el patrón de distribución temporal de la lluvia para los eventos agrupados por cuartil. Las probabilidades de ocurrencia de los eventos es mayor para los cuartiles I y II (84\% juntos), apenas 12\% para el cuartil III y solo $2 \%$ para el cuartil IV. La Tabla 4 muestra los coeficientes de distribución temporal de la tormenta para cada cuartil.

Tabla 4: Patrón de distribución de la lluvia para $\mathrm{Td}<=2 \mathrm{~h}$ y $\mathrm{P}>=30 \mathrm{~mm}$.

\begin{tabular}{ccccc}
\hline \multirow{2}{*}{ \% T acum } & C-I & C-II & C-III & C-IV \\
\cline { 2 - 5 } & \% P Acum & \% P Acum & \% P Acum & \% P Acum \\
\hline 0 & 0 & 0 & 0 & 0 \\
5 & 4.5 & 2.2 & 0.9 & 1.2 \\
10 & 13.5 & 6.5 & 2.6 & 3.2 \\
15 & 22.8 & 11.5 & 5.3 & 5.7 \\
20 & 33.1 & 17.3 & 8.3 & 8.3 \\
25 & 42.9 & 23.8 & 11.4 & 11.0 \\
30 & 51.4 & 31.4 & 15.9 & 14.5 \\
35 & 58.2 & 39.9 & 20.9 & 18.3 \\
40 & 64.7 & 49.2 & 26.3 & 22.3 \\
45 & 70.8 & 58.2 & 33.3 & 26.4 \\
50 & 75.7 & 66.8 & 42.3 & 30.9 \\
55 & 79.5 & 73.6 & 51.1 & 35.9 \\
60 & 83.1 & 79.3 & 58.9 & 42.4 \\
65 & 86.4 & 84.2 & 66.3 & 49.5 \\
70 & 89.5 & 88.2 & 73.3 & 57.0 \\
75 & 92.0 & 91.5 & 80.1 & 65.0 \\
80 & 94.0 & 94.2 & 86.1 & 73.5 \\
85 & 95.8 & 96.3 & 91.6 & 84.4 \\
90 & 97.4 & 97.7 & 95.9 & 91.7 \\
95 & 98.7 & 98.9 & 98.5 & 97.4 \\
100 & 100.0 & 100.0 & 100.0 & 100.0 \\
\hline
\end{tabular}

La Figura 11 muestra para cada cuartil las curvas de distribución promedio de la lluvia en el tiempo, y las Figuras 12, 13, 14 y 15 muestra los hietogramas desarrollados para cada cuartil, cada uno ilustra el porcentaje de la lluvia total para incrementos del $10 \%$ de la duración de la tormenta.

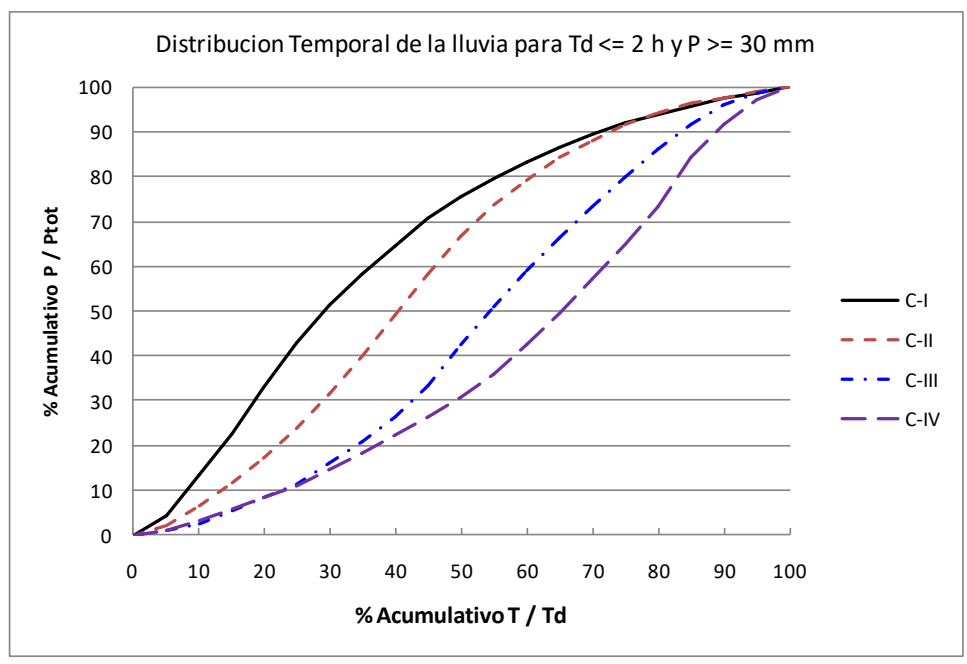

Figura 11: Curvas de distribución temporal de la lluvia para $\mathrm{Td}<=2 \mathrm{~h}$ y $\mathrm{P}>=30 \mathrm{~mm}$ 


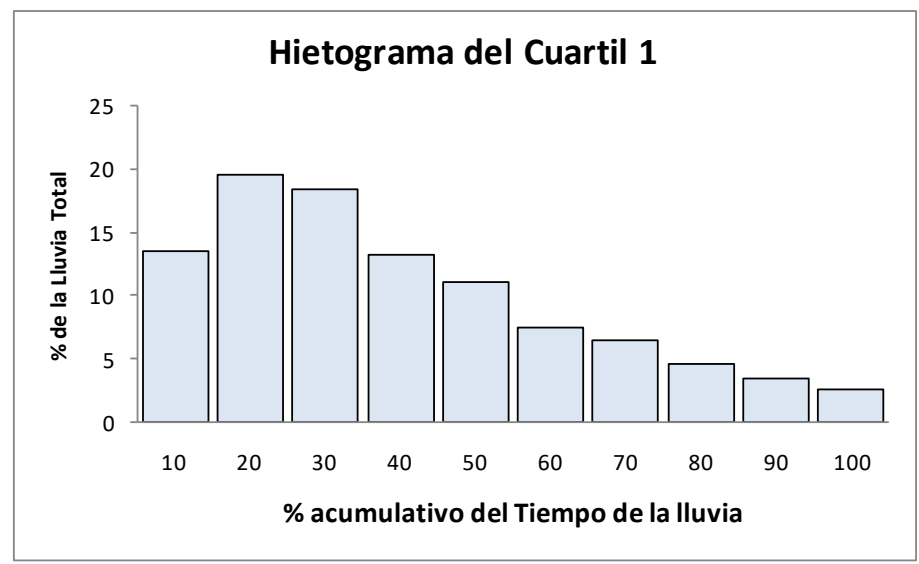

Figura 12: Hietograma del Cuartil I para $\mathrm{Td}<=2 \mathrm{~h}$ y $\mathrm{P}>=30 \mathrm{~mm}$

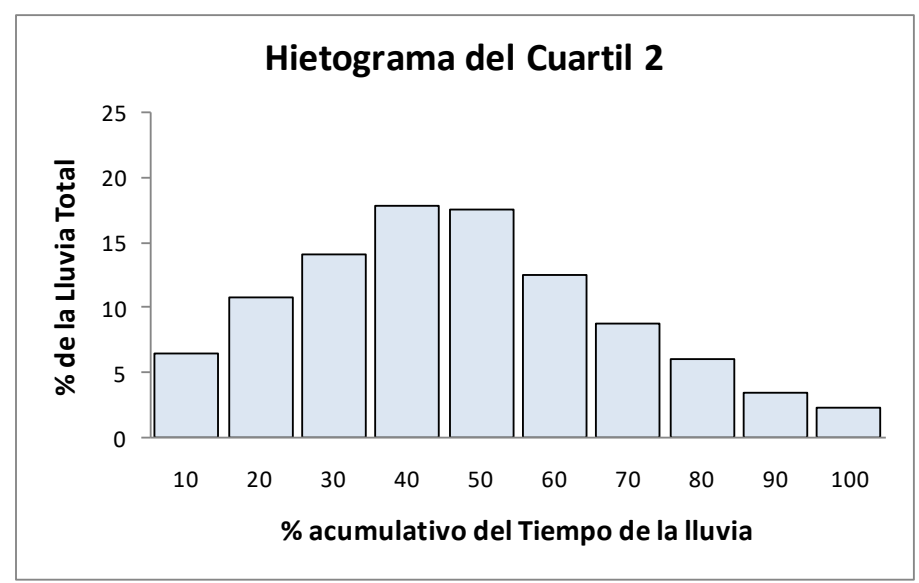

Figura 13: Hietograma del Cuartil II para $\mathrm{Td}<=2 \mathrm{~h}$ y P $>=30 \mathrm{~mm}$

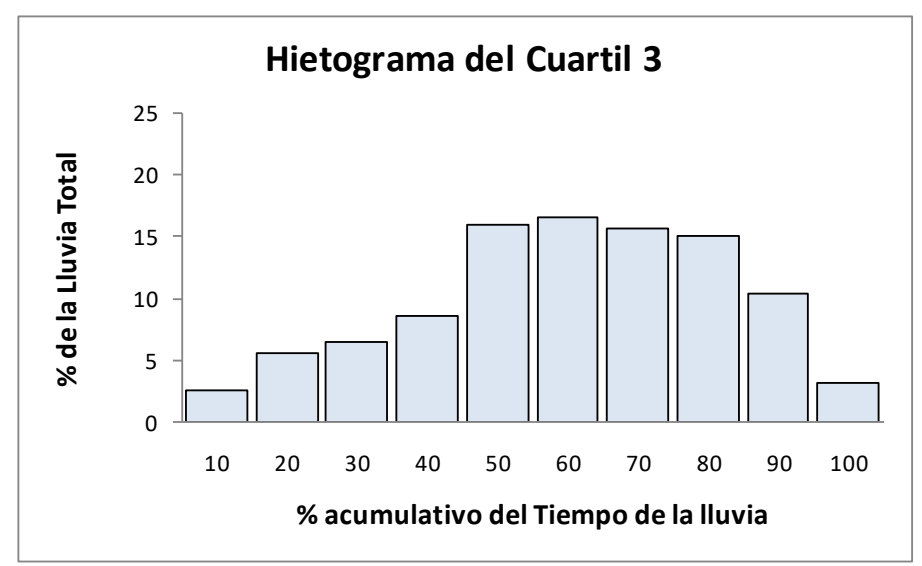

Figura 14: Hietograma del Cuartil III para $\mathrm{Td}<=2 \mathrm{~h}$ y $\mathrm{P}>=30 \mathrm{~mm}$ 


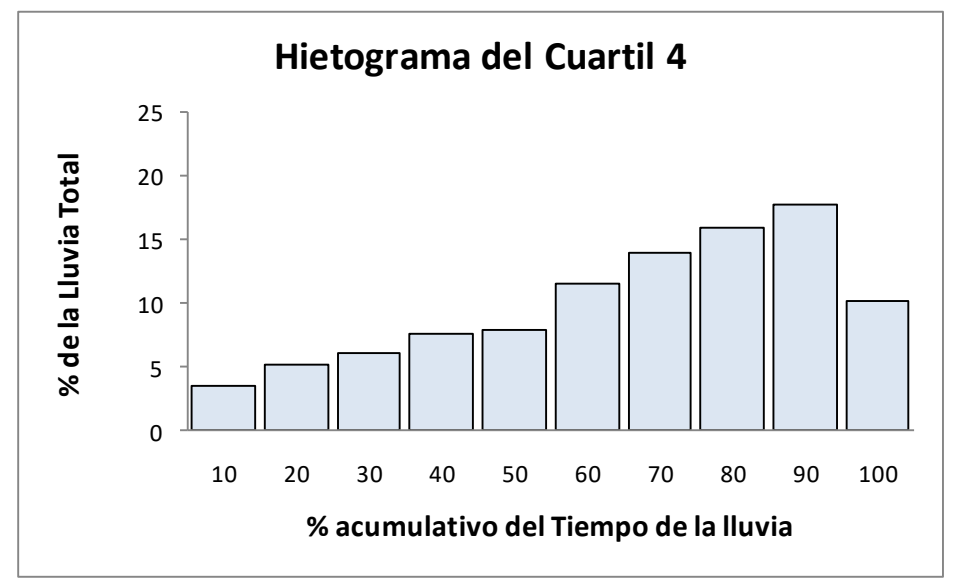

Figura 15: Hietograma del Cuartil IV para $\mathrm{Td}<=2 \mathrm{~h}$ y $\mathrm{P}>=30 \mathrm{~mm}$

De los hietogramas anteriores se obtiene los coeficientes de avance de las tormentas " $r$ ", para el cuartil I es 0.25 , para el cuartil II es 0.40 , para el cuartil III es 0.60 y para el cuartil IV es 0.90 .

\subsection{Resultados y Análisis de Resultados}

Si se comparan los valores de las Tablas 2 y 4, correspondientes a los patrones de distribución de la lluvia para eventos mayores que $20 \mathrm{~mm}$ y $30 \mathrm{~mm}$ respectivamente, no hay mucha diferencia entre ellos, por ejemplo, para un tiempo acumulado del $50 \%$, en el cuartil I, el \% acumulado de altura de precipitación es $77.8 \%(\mathrm{P}>20 \mathrm{~mm})$ y $75.7 \%(\mathrm{P}>30 \mathrm{~mm})$, en el cuartil II, el \% acumulado de altura de precipitación es $66.3 \%(\mathrm{P}>20 \mathrm{~mm})$ y $66.8 \%(\mathrm{P}>30 \mathrm{~mm})$, en el cuartil III, el \% acumulado de altura de precipitación es $40.4 \%(\mathrm{P}>20 \mathrm{~mm})$ y $42.3 \%(\mathrm{P}>30 \mathrm{~mm})$, y en el cuartil IV, el \% acumulado de altura de precipitación es $30.9 \%(\mathrm{P}>20 \mathrm{~mm})$ y $30.9 \%(\mathrm{P}>30 \mathrm{~mm})$.

Probablemente esto se deba a que los eventos entre $20 \mathrm{~mm}$ y $30 \mathrm{~mm}$ corresponden a los eventos más probables de los eventos analizados (ver figuras 2 y 9), y por eso tienen mayor peso a la hora obtener los hietogramas promedios.

Los eventos de lluvia predominantes ocurren en los cuartiles I y II, con un promedio de $83 \%$ de probabilidad de ocurrencia, con respecto al total de eventos analizados en cada caso.

\section{CONCLUSIONES}

En base al análisis de los datos de lluvia de la estación Aeropuerto de Managua, se pueden delinear las siguientes conclusiones:

Para las tormentas analizadas, con altura de precipitación mayor que 20 y $30 \mathrm{~mm}$, y duración menor o igual a dos horas, el patrón de distribución temporal de la lluvia de corta duración en la estación Aeropuerto de Managua, es asimétrico.

El perfil de la lluvia predominante es del cuartil II, con un probabilidad de ocurrencia de $50 \%$, seguido del cuartil I (31\%), el cuartil III (12\%), y el cuartil IV con un probabilidad de apenas $3 \%$.

El coeficiente de avance de la tormenta, para el cuartil I es aproximadamente $r=0.2$, y para el cuartil II es aproximadamente $r=0.4$, por lo que es recomendable utilizar los hietogramas adimensionales de estos 
cuartiles para la construcción de los hietogramas de diseño en la subcuenca sur del Lago Xolotlán, donde las microcuencas tienen aproximadamente un tiempo de concentración menor o igual a dos horas.

\section{REFERENCIAS}

Balbastre, R., Garcia, R., y Domenech, I. (2018). Análisis comparativo de metodologías de cálculo de tormentas de diseño para su aplicación en hidrología urbana. [Tesis de Maestria, Universidad Politécnica de Valencia]. https://riunet.upv.es/bitstream/handle/10251/100090/BALBASTRE\%20\%20An\%C3\%A1lisis\%20comparativo\%20de\%20metodolog\%C3\%ADas\%20de\%20c\%C3\%A1lculo\%20 de\%20tormentas\%20de\%20dise\%C3\%B10\%20para\%20su\%20aplica....pdf?sequence $=1$

Back, A.J. (2011). Time distribution of heavy rainfall events in Urussanga, Santa Catarina State, Brazil. Acta Scientiarum. Agronomy. Maringá, v. 33, n. 4, p. 583-588. DOI: 10.4025/actasciagron.v33i4.6664

Chow, V.T., Maidment, D. \&Mays, L. (1988). Applied Hydrology. McGraw Hill.

Huff, F. (1967). Time distribution of rainfall in heavy storms. Water Resources Research, 3(4), pp. 1007 1019. https://doi.org/10.1029/WR003i004p01007

Instituto Nicaragüense de Estudios Territoriales, Autoridad Nacional del Agua, Universidad Nacional de Ingeniería, Programa de Asistencia Técnica en Agua y Saneamiento. (2014).Cuencas hidrográficas de Nicaragua bajo la metodología Pfafstetter. Managua: Autor.

Mays, L. W. (2001). Stormwater collection systems design handbook. Hydrology for drainage system design and analysis, Chapter 4.AccessEngineering: McGraw-Hill Professional.

Natural Resources Conservation Services, NRCS. (1986). Urban hydrology for small watersheds TR-55. Conservation Engineering Division Technical Release 55. Second Edition.https://www.nrcs.usda.gov/Internet/FSE DOCUMENTS/stelprdb1044171.pdf

Watt, W. E., Chow, K. C. A., Hogg, W. D. y Lathem, K. W., 1986. A 1-h urban design storm for Canada. Canadian Journal of Civil Engineering, 13(3), pp. 293-300.https://doi.org/10.1139/186-041

\section{SEMBLANZA DE LOS AUTORES}

Miguel E. Blanco Chávez: Obtuvo el grado de Ingeniero Civil en la Universidad Nacional de Ingeniería, Nicaragua. Desarrolló sus estudios de Maestría en Ingeniería Ambiental en el Programa de Investigación y Docencia en Medio Ambiente (PIDMA) de la UNI, Nicaragua. Ha participado como consultor en proyectos hidroeléctricos, de amenazas por inundación, de drenaje pluvial y manejo de cuencas hidrográficas. Ha sido profesor de hidráulica e hidrología en la carrera de ingeniería civil en la FTC de la UNI. Actualmente imparte los cursos Hidrología Aplicada y Redes de Alcantarillado en el Programa de Investigación, Estudios Nacionales y Servicios del Ambiente (PIENSA) de la UNI, Nicaragua. 